Research Paper

\title{
HE4 and elF3a Expression Correlates with Surgical Outcome and Overall Survival in Ovarian Cancer Patients with Secondary Cytoreduction
}

\author{
Chen-Hui Luo, ${ }^{1,2}$, Min Zhao'2, Xiao-Yan Chen³, Shohreh Shahabi', Wenan Qiang5, Liang Zeng6, Jing \\ Wang ${ }^{\boxplus 凶}$, Hong-Hao Zhou ${ }^{2}$
}

1. Laboratory Animal Research Center, Hunan Cancer Hospital, the Affiliated Cancer Hospital of Xiangya School of Medicine, Central South University, Changsha, Hunan, People's Republic of China.

2. Department of Clinical Pharmacology, Institute of Clinical Pharmacology, Hunan Key Laboratory of Pharmacogenetics, Xiangya Hospital, Central South University, Changsha, Hunan, People's Republic of China.

3. Department of Pathology, Hunan Cancer Hospital, the Affiliated Cancer Hospital of Xiangya School of Medicine, Central South University, Changsha, Hunan, People's Republic of China.

4. Department of Obstetrics and Gynecology, Division of Gynecologic Oncology, Prentice Women's Hospital, Northwestern University Feinberg School of Medicine, Chicago, IL, United States.

5. Center for Developmental Therapeutics, Chemistry of Life Processes Institute, Department of Obstetrics and Gynecology-Division of Reproductive Science in Medicine, Northwestern University Feinberg School of Medicine, Chicago, IL, United States.

6. Department of Pathology, Guangzhou Women and Children's Medical Center, Guangzhou Medical University, Guangzhou, Guangdong, People's Republic of China.

7. Department of Gynecologic Oncology, Hunan Cancer Hospital, the Affiliated Cancer Hospital of Xiangya School of Medicine, Central South University, Changsha, Hunan, People's Republic of China.

$\square$ Corresponding authors: Hong-Hao Zhou, MD, Institute of Clinical Pharmacology, Xiangya Hospital, Central South University, 110 Xiangya Road, Changsha 410008, Hunan, People's Republic of China (phone number: +86 7318480 5379, E-mail: hhzhou2003@163.com); and Jing Wang, MD, PhD, Department of Gynecologic Oncology, Hunan Cancer Hospital, The Affiliated Cancer Hospital of Xiangya School of Medicine, Central South University, 582 Xianjiahu Road, Changsha, 410013, Hunan, People's Republic of China (phone number: +86 7318865 1849, E-mail: wangjing189@163.com).

(c) Ivyspring International Publisher. This is an open access article distributed under the terms of the Creative Commons Attribution (CC BY-NC) license (https://creativecommons.org/licenses/by-nc/4.0/). See http://ivyspring.com/terms for full terms and conditions.

Received: 2018.01.28; Accepted: 2018.04.28; Published: 2018.06.14

\begin{abstract}
For recurrent ovarian cancer (ROC), secondary cytoreductive surgery (SCS) is recommended as one optional treatment. However, little is known about the expression and clinical significance of biomarkers during SCS. Human epididymis protein 4 (HE4) is a clinical biomarker for ovarian cancer. Eukaryotic translation initiation factor $3 a$ (elF3a) is investigated extensively as a potential biomarker for malignancy. The purpose of this study was to investigate the expressions of HE4 and elF3a at SCS, as well as their associations with surgical outcome and survival in ROC patients. Immunohistochemistry was performed to determine the expressions of HE4 and elF3a in ovarian tumors taken from both initial and secondary cytoreductive surgery of 35 ROC patients. elF3a levels were significantly increased at SCS, compared to those at initial cytoreductive surgery (ICS), while HE4 levels were similar. Both HE4 and elF3a expressions were associated with surgical outcome, in terms of residual tumor. For ICS, patients with high HE4 expression achieved a higher incidence of optimal cytoreduction than those with low HE4 expression $(81.0 \%$ vs. $33.3 \%, P=0.015)$. A similar result happened in SCS, indicated by higher incidence of no residual tumor in patients with high HE4 expression $(76.4 \%$ vs. $44.4 \%, \mathrm{P}=0.046)$. And high HE4 expression at SCS was more likely to enhance surgical outcome of SCS $(77.8 \%$ vs. $29.4 \%, P=0.038)$. Therefore, high HE4 expression at either surgery is a predictor of better overall survival $(O S)(P=0.011$ and 0.002). Furthermore, patients with an elevated total score (TS) of HE4 between the two surgeries tended to have prolonged OS, compared to those with a non-elevated TS of HE4 $(P=0.076)$. For elF3a, initial elF3a expression was associated with secondary residual tumor $(P=0.035)$, and the difference in elF3a expression between the two surgeries correlated with OS $(P=0.052)$. The expressions of HE4 and elF3a in tumor specimens correlated with surgical outcome and predicted OS in ROC patients with SCS, thus meriting further investigation.
\end{abstract}

Key words: HE4, eIF3a, recurrent ovarian cancer (ROC), secondary cytoreductive surgery (SCS), residual tumor, overall survival 


\section{Introduction}

Despite advances in the development of novel treatment methods, epithelial ovarian cancer (EOC) is still the leading cause of death from gynecologic cancer[1, 2]. Overall, $85 \%$ of ovarian cancer patients will have recurrent disease[3]. Secondary cytoreductive surgery (SCS) is a recommended optional treatment for recurrent ovarian cancer (ROC)[1]. However, only a small proportion of patients actually undergo SCS. Little is known about the expression and clinical significance of biomarkers in tumor specimens taken from ROC during SCS.

Among the biomarkers for EOC, human epididymis protein 4 (HE4) is regarded as one of the most promising serum biomarkers[4], because numerous studies have investigated its role in the diagnosis, prognosis, and follow-up of EOC and found that HE4 is a more valuable serum biomarker than CA125, currently a routine biomarker in the diagnosis of EOC and its recurrence[5-7]. Previous studies have also reported that high serum levels of HE4 correlate with both chemoresistance and a decrease in survival of women with $\mathrm{EOC}[8,9]$. However, to our knowledge, no reports have examined the expression of HE4 in ROC specimens taken from SCS. So we selected HE4 as a target biomarker in the present study.

Eukaryotic translation initiation factor 3a (eIF3a) is the core subunit of the eIF3 complex, which is crucial for the initiation of mRNA translation, and links translation initiation to transcription[10, 11], to mRNA export[12], and to the nonsense-mediated decay pathway[13]. eIF3a is under extensive investigation as a potential prognostic biomarker for different malignancies[14-18]. We previously presented a pilot study on the increased expression of eIF3a in primary EOC tumors, and a positive correlation between eIF3a and HE4[17, 19]. Consequently, the expression of eIF3a in tumor specimens from SCS has aroused our interest.

In the present study we used immunohistochemistry (IHC) to investigate the expressions of HE4 and eIF3a in women with ROC who underwent SCS. Furthermore, we evaluated the relationships between these biomarkers and clinicopathological parameters and survival of women with ROC.

\section{Materials and Methods}

\section{Patients and specimens}

To obtain tumor specimens, we screened all ovarian cancer patients at the Hunan Cancer Hospital (Changsha, China) from January 2004 to December 2014. After applying our inclusion and exclusion criteria we were left with 35 patients. Our inclusion criteria were: (a) all patients had undergone both initial cytoreductive surgery (ICS) and SCS at the same hospital; (b) all specimens were confirmed histologically as epithelial ovarian carcinoma by a pathologist with expertise in gynecologic cancer. Our exclusion criteria included metastatic or non-epithelial ovarian tumors. All patients were staged according to the International Federation of Gynecology and Obstetrics (FIGO) surgical staging system. Tumor specimens and clinical data were collected from the Department of Pathology, Hunan Cancer Hospital, as approved by the Ethics Committee of Hunan Cancer Hospital (Changsha, China).

\section{IHC detection}

The procedure used in this study was described previously[17, 19]. Tumor sections (4 $\mu \mathrm{m}$ thick) were prepared from paraffin samples. After deparaffinization and rehydration, IHC was performed using an UltraSensitiveTM SP IHC Kit (Fuzhou Maixin) and a DAB Detection Kit (Streptavidin-Biotin, Fuzhou Maixin), following the protocol recommended by the manufacturer. The primary antibodies were anti-HE4 recombinant rabbit monoclonal antibody (1:1000, Abcam, ab109298) and anti-eIF3a recombinant rabbit monoclonal antibody (1:1000, Abcam, ab128996). Slides were assessed by two independent experienced pathologists blinded to the patient's status. In the case of an inconsistent score between them, a third pathologist was consulted for the final evaluation. Staining intensity was scored from 0 to 3 ( 0 , negative; 1 , weak; 2 , moderate; 3 , strong). The heterogeneity of staining was scored from 0 to 3 , depending on the percentage of positively stained tumor cells $(\mathbf{0}, 0-25 \% ; \mathbf{1}, 25-50 \% ; \mathbf{2}, 50-75 \%$; $\mathbf{3}$, $75-100 \%)$. The intensity and heterogeneity scores were multiplied to generate the total score (TS). TS >= 5 was considered as a high expression of HE4, while TS $<5$ was a low expression of HE4. In the case of eIF3a, TS $>=4$ was considered as high expression, while TS $<4$ was low expression.

\section{Statistical analysis}

The data are presented as mean \pm standard deviation. The paired t-test, McNemar's test, ANOVA test, Student's t-test, chi-square test, and multivariate Cox proportional hazard regression were performed as appropriate using SPSS 22.0 software. Overall survival (OS) was defined as the interval in months between the initial EOC resection and either death or the last observation. Progression free survival (PFS) was defined as the number of months from the ICS to the diagnosis of relapse. Patients that were alive at the 
end of follow-up were censored. OS and PFS were evaluated using the Kaplan-Meier method and log-rank test. Figures were made with Graph-Pad Prism 6.0. All p-values were two-tailed. $\mathrm{P}<0.05$ was considered to be significant.

\section{Results}

\section{Clinicopathologic variables of patients}

The cohort included 35 women with ROC, but the clinical data of two patients were missing. The median age of patients at the time of diagnosis was 49 years old and ranged from 29 to 70 years old. All patients did not receive radiotherapy or biological therapy, neither before nor after ICS, but some of them received platinum/taxane-based neoadjuvant chemotherapy (NACT). The postoperative chemotherapy they received was also a platinum/ taxane-based chemotherapy. There were also two patients lacking the information on recurrence date. Therefore, Out of the 31 patients, 26 were platinum sensitive (26/31, 83.9\%). The clinical and pathological information of each patient is shown in Table 1.

\section{Expressions of HE4 and elF3a at initial and secondary cytoreductive surgery}

The expressions of HE4 and eIF3a for each patient are listed in Table 1. Figure 1A shows representative HE4 and eIF3a staining. The results of the paired t-test and McNemar's test both indicate that HE4 levels were similar between ICS and SCS while eIF3a levels were significantly increased at time of SCS (Figure 1B).

For ovarian cancer, residual tumor $<1 \mathrm{~cm}$ defines an optimal cytoreduction and fine surgical outcome (Table 2, group $0 \mathrm{~cm}$ plus group $<1 \mathrm{~cm}$ )[1]. Conversely, residual tumor $>1 \mathrm{~cm}$ means a suboptimal cytoreduction and poor surgical outcome. In the present study, residual tumor was associated with the expressions of both HE4 and eIF3a in specimens from ICS and SCS.

Table 1. Details of each patient.

\begin{tabular}{|c|c|c|c|c|c|c|c|c|c|c|c|c|c|c|c|c|c|c|}
\hline No. & $\begin{array}{l}\text { TS of } \\
\text { HE4 (I) }\end{array}$ & $\begin{array}{l}\text { TS of HE4 } \\
\text { (II) }{ }^{a}\end{array}$ & $\begin{array}{l}\text { TS of eIF3a } \\
\text { (I) a }\end{array}$ & $\begin{array}{l}\text { TS of eIF3a } \\
\text { (II) a }\end{array}$ & $\begin{array}{l}\text { RT } \\
(\mathrm{I})^{\mathrm{a}} \\
(\mathrm{cm})\end{array}$ & $\begin{array}{l}\text { RT } \\
(\mathrm{II})^{\mathrm{a}} \\
(\mathrm{cm})\end{array}$ & $\begin{array}{l}\text { Meno } \\
\text { pause }\end{array}$ & $\begin{array}{l}\text { Ascites } \\
(\mathrm{ml})\end{array}$ & $\begin{array}{l}\text { NAC } \\
\mathrm{T}\end{array}$ & Grade & $\begin{array}{l}\text { Subty } \\
\text { pe }^{\text {b }}\end{array}$ & Stage & LN-M & $\begin{array}{l}\text { Cycles } \\
\text { (I) a }\end{array}$ & $\begin{array}{l}\text { Cycles } \\
\text { (II) }\end{array}$ & $\begin{array}{l}\text { PFS } \\
\text { (months) }\end{array}$ & $\begin{array}{l}\text { OS } \\
\text { (months) }\end{array}$ & $\begin{array}{l}\text { OS } \\
\text { statusc }^{c}\end{array}$ \\
\hline 1 & 6 & 4.5 & 2 & 6 & $>1$ & 0 & $\mathrm{NO}$ & 1000 & $\mathrm{NO}$ & UNK & $S$ & III & Neg & 8 & 4 & 14.8 & 62.8 & 1 \\
\hline 2 & 6 & 6 & 2 & 6 & NA & NA & NA & NA & NA & NA & NA & NA & NA & NA & NA & NA & NA & NA \\
\hline 3 & 6 & 6 & 4 & 2 & $>1$ & $>1$ & YES & 2500 & $\mathrm{NO}$ & G2 & $S$ & III & Pos & 3 & 32 & 42.8 & 111.8 & 0 \\
\hline 4 & 4.5 & NA & 0 & 2 & $>1$ & 0 & $\mathrm{NO}$ & 0 & $\mathrm{NO}$ & BOL & $\mathrm{M}$ & III & Neg & 2 & 5 & 5.8 & 29.1 & 1 \\
\hline 5 & 6 & 7.5 & 1 & 6 & UNK & 0 & YES & 0 & NO & BOL & $\mathrm{M}$ & UNK & Neg & 3 & 4 & 66.3 & 104.9 & 1 \\
\hline 6 & 6 & NA & 2 & 4 & 0 & $<1$ & $\mathrm{NO}$ & 0 & YES & G3 & $\mathrm{O}$ & III & Neg & 9 & UNK & 14.9 & 54.2 & 1 \\
\hline 7 & 3 & 4.5 & 1 & 2 & $>1$ & $<1$ & $\mathrm{NO}$ & 0 & NO & G3 & $\mathrm{O}$ & III & UNK & 16 & 2 & 13.5 & 28.3 & 1 \\
\hline 8 & 6 & 9 & 2 & 6 & $<1$ & 0 & $\mathrm{NO}$ & 5000 & $\mathrm{NO}$ & BOL & $\mathrm{M}$ & III & Neg & 6 & 6 & 6.1 & 123 & 0 \\
\hline 9 & 6 & 6 & 2 & 4 & 0 & 0 & YES & 1000 & $\mathrm{NO}$ & G3 & $\mathrm{O}$ & IV & Neg & 6 & 7 & 55.7 & 89.7 & 1 \\
\hline 10 & 4.5 & 6 & 4 & 4 & $<1$ & 0 & $\mathrm{NO}$ & 5000 & $\mathrm{NO}$ & G2 & $S$ & III & Neg & 14 & 3 & 25.5 & 32.5 & 1 \\
\hline 11 & 4.5 & 4.5 & 0 & 1 & $>1$ & $<1$ & YES & 4800 & NO & G3 & $S$ & III & UNK & 2 & UNK & UNK & 92.6 & 0 \\
\hline 12 & 4.5 & NA & 0 & 6 & $>1$ & $<1$ & $\mathrm{NO}$ & 3000 & $\mathrm{NO}$ & G2 & $\mathrm{O}$ & III & UNK & 5 & 3 & UNK & 47.9 & 0 \\
\hline 13 & 3 & 3 & 1 & 2 & 0 & 0 & $\mathrm{NO}$ & 0 & NO & G3 & $\mathrm{O}$ & III & Pos & 11 & 4 & 14.9 & 36 & 1 \\
\hline 14 & 3 & 3 & 2 & 3 & $<1$ & 0 & YES & 3800 & $\mathrm{NO}$ & G2 & S & IV & UNK & 7 & 4 & 17.8 & 31.4 & 1 \\
\hline 15 & 2 & 1.5 & 1 & 3 & $>1$ & 0 & YES & 3000 & NO & G2 & $S$ & III & Pos & 8 & 8 & 39.1 & 87 & 0 \\
\hline 16 & 4.5 & 4.5 & 3 & 4 & $>1$ & $<1$ & YES & 2500 & $\mathrm{NO}$ & G2 & $\mathrm{O}$ & IV & UNK & 3 & 14 & 45.5 & 63 & 1 \\
\hline 17 & NA & NA & 0 & 0 & $>1$ & 0 & YES & 7000 & NO & BOL & $\mathrm{M}$ & UNK & UNK & 0 & UNK & 8.9 & 46. & 1 \\
\hline 18 & 5 & NA & 0 & 0 & 0 & 0 & $\mathrm{NO}$ & 0 & YES & G2 & $\mathrm{O}$ & IV & Neg & 5 & 6 & 17.9 & 137.5 & 1 \\
\hline 19 & NA & 4.5 & 0 & 0 & $<1$ & $<1$ & YES & 0 & YES & UNK & $\mathrm{O}$ & IV & Neg & 4 & 5 & 30.3 & 49.3 & 1 \\
\hline 20 & 9 & 9 & 4 & 0 & 0 & 0 & $\mathrm{NO}$ & 100 & $\mathrm{NO}$ & UNK & $\mathrm{O}$ & I & Neg & 2 & 4 & 33.5 & 85.9 & 0 \\
\hline 21 & 9 & 9 & 2 & 0 & 0 & 0 & $\mathrm{NO}$ & 0 & YES & BOL & S & III & Neg & 2 & UNK & 35 & 77.2 & 0 \\
\hline 22 & 9 & 6 & 0 & 6 & 0 & 0 & $\mathrm{NO}$ & 3000 & $\mathrm{NO}$ & BOL & $S$ & III & Neg & 6 & 7 & 43.3 & 82.7 & 0 \\
\hline 23 & 7.5 & 4.5 & NA & NA & $>1$ & $<1$ & $\mathrm{NO}$ & 0 & $\mathrm{NO}$ & G3 & $\mathrm{O}$ & III & UNK & 16 & 2 & 13.5 & 28.3 & 1 \\
\hline 24 & 6 & 7.5 & 2 & 4 & 0 & 0 & $\mathrm{NO}$ & 100 & $\mathrm{NO}$ & G3 & $\mathrm{O}$ & I & Neg & 8 & 11 & 26.3 & 85.9 & 0 \\
\hline 25 & 9 & 7.5 & 4 & 0 & 0 & 0 & $\mathrm{NO}$ & 2500 & $\mathrm{NO}$ & G2 & $\mathrm{O}$ & III & Pos & 6 & 11 & 9.8 & 48.1 & 1 \\
\hline 26 & 7.5 & 7.5 & 4 & NA & 0 & 0 & YES & 0 & $\mathrm{NO}$ & G2 & $\mathrm{O}$ & I & Neg & 4 & 3 & 29.5 & 75 & 0 \\
\hline 27 & 7.5 & NA & 1 & NA & 0 & 0 & $\mathrm{NO}$ & 400 & $\mathrm{NO}$ & BOL & $S$ & UNK & UNK & 0 & UNK & 39.7 & UNK & UNK \\
\hline 28 & 7.5 & 6 & 2 & 1 & $<1$ & UNK & YES & 3000 & $\mathrm{NO}$ & G2 & $\mathrm{O}$ & III & Pos & 9 & 2 & 20.5 & 94 & 0 \\
\hline 29 & 7.5 & 6 & NA & NA & 0 & $>1$ & $\mathrm{NO}$ & 4500 & $\mathrm{NO}$ & BOL & $\mathrm{M}$ & UNK & UNK & 2 & 5 & 70.3 & 81.6 & 1 \\
\hline 30 & 6 & 7.5 & 1 & 2 & 0 & $<1$ & YES & 2500 & YES & G2 & $\mathrm{O}$ & III & Neg & 14 & UNK & 10.9 & 141.7 & 1 \\
\hline 31 & 6 & 6 & 0 & 0 & 0 & 0 & YES & 0 & $\mathrm{NO}$ & G2 & $S$ & I & Neg & 7 & 14 & 35.6 & 84.8 & 1 \\
\hline 32 & 6 & 7.5 & 1 & 1 & $>1$ & $<1$ & $\mathrm{NO}$ & 150 & $\mathrm{NO}$ & G2 & $\mathrm{O}$ & III & UNK & 9 & 20 & 33.6 & 127.8 & 1 \\
\hline 33 & 7.5 & 9 & 2 & 1 & 0 & 0 & YES & 0 & $\mathrm{NO}$ & BOL & $\mathrm{O}$ & II & Neg & 3 & 4 & 43.6 & 150.5 & 1 \\
\hline 34 & 7.5 & 9 & 2 & 2 & $<1$ & 0 & YES & 6000 & $\mathrm{NO}$ & G2 & S & III & Neg & 8 & 6 & 49.2 & 131.9 & 0 \\
\hline 35 & 7.5 & 6 & NA & NA & NA & NA & NA & NA & NA & NA & NA & NA & NA & NA & NA & NA & NA & NA \\
\hline \multicolumn{19}{|c|}{$\begin{array}{l}\text { Abbr.: TS: total score; RT: residual tumor; NACT: neoadjuva } \\
\text { free survival; OS: overall survival; NA: not available; UNK: } \\
\text { a: (I) for the initial surgery; (II) for the secondary surgery; } \\
\text { b: M=Mucinous, S=Serous, O=Others; }\end{array}$} \\
\hline
\end{tabular}


A
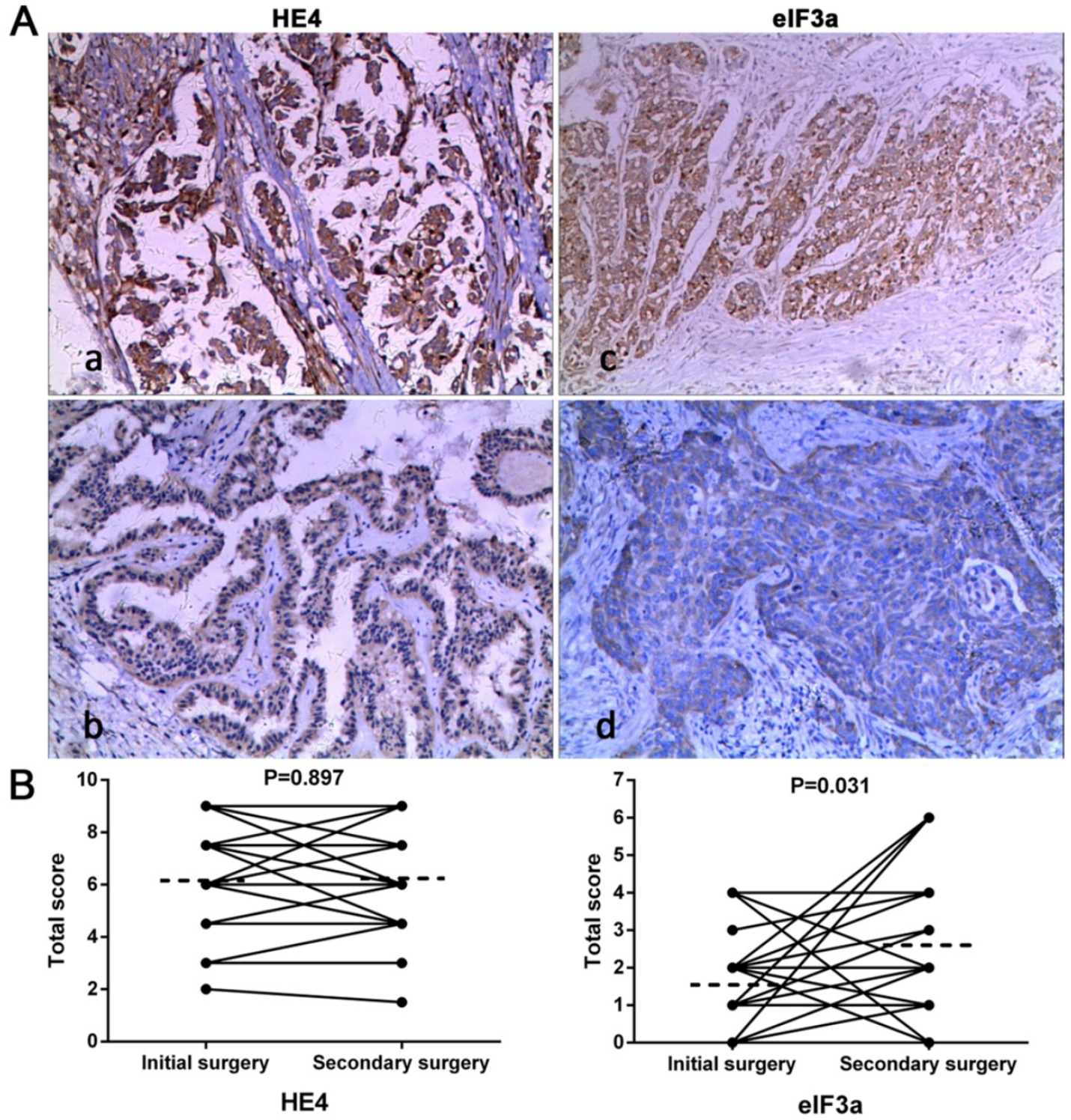

Figure 1. HE4 and elF3a expressions in women with ROC. (A) The representative results of HE4 and elF3a staining in IHC at 100x magnification. (a) and (c) High expression, (b) and (d) Low expression. (B) Comparisons of HE4 and elF3a levels between initial and secondary surgery by paired t-test, respectively. Dashed lines indicate the mean TS of each group. Initial surgery vs. secondary surgery: 6.17 vs. 6.21 for HE4, 1.57 vs. 2.6 for elF3a.

Table 2. Comparisons of HE4 and elF3a expression among groups of residual tumor size post-surgery, respectively.

\begin{tabular}{|c|c|c|c|c|c|c|c|c|c|c|}
\hline & & & \multicolumn{8}{|c|}{ Residual tumor } \\
\hline & & & \multicolumn{4}{|c|}{ Initial surgery, $\mathrm{n}(\%)$} & \multicolumn{4}{|c|}{ Secondary surgery, n (\%) } \\
\hline & & & $0 \mathrm{~cm}$ & $<1 \mathrm{~cm}$ & $>1 \mathrm{~cm}$ & $P$ value & $0 \mathrm{~cm}$ & $<1 \mathrm{~cm}$ & $>1 \mathrm{~cm}$ & $P$ value \\
\hline \multirow[t]{4}{*}{ HE4 } & Initial Surgery & Low expression & $1(11.1 \%)$ & $2(22.2 \%)$ & $6(66.7 \%)$ & 0.015 & $5(55.6 \%)$ & $4(44.4 \%)$ & 0 & 0.270 \\
\hline & & High expression & $14(66.7 \%)$ & $3(14.3 \%)$ & $4(19.0 \%)$ & & $15(71.5 \%)$ & $4(19.0 \%)$ & $2(9.5 \%)$ & \\
\hline & Secondary Surgery & Low expression & -- & -- & -- & no use & $4(44.4 \%)$ & $5(55.6 \%)$ & 0 & 0.046 \\
\hline & & High expression & -- & -- & -- & & $13(76.4 \%)$ & $2(11.8 \%)$ & $2(11.8 \%)$ & \\
\hline \multirow[t]{4}{*}{ eIF3a } & Initial Surgery & Low expression & $11(44.0 \%)$ & $5(20.0 \%)$ & $9(36.0 \%)$ & 0.760 & $17(68.0 \%)$ & $8(32.0 \%)$ & 0 & 0.035 \\
\hline & & High expression & $3(60.0 \%)$ & $1(20.0 \%)$ & $1(20.0 \%)$ & & $4(80.0 \%)$ & 0 & $1(20.0 \%)$ & \\
\hline & Secondary Surgery & Low expression & -- & -- & -- & no use & $11(61.1 \%)$ & $6(33.3 \%)$ & $1(5.6 \%)$ & 0.660 \\
\hline & & High expression & -- & -- & -- & & $8(72.7 \%)$ & $3(27.3 \%)$ & 0 & \\
\hline
\end{tabular}

a: analysis with chi-square test.

For the initial surgery, patients with high HE4 expression achieved a higher incidence of optimal cytoreduction than those with low HE4 expression (Table 2, $\mathrm{P}=0.015$ ). A similar result happened in SCS, as indicated by the higher incidence of no residual tumor in patients with high HE4 expression (Table 2,
$P=0.046)$. HE4 expression at initial surgery was not associated with residual tumor after SCS (Table 2, P = 0.270), which means that HE4 expression at the time of the initial surgery cannot be used to predict the surgical outcome of SCS. However, HE4 expression at SCS was associated with the variation in residual 
tumor between the two surgeries. Patients with high HE4 expression at SCS were more likely to enhance their surgical outcome of SCS (Table 3, 77.8\% vs. $29.4 \%, \mathrm{P}=0.038$ ).

For eIF3a, initial eIF3a expression was not associated with initial residual tumor (Table 2, $\mathrm{P}=$ 0.760 ) but was associated with secondary residual tumor (Table 2, $\mathrm{P}=0.035$ ). This was caused by all nine patients with low eIF3a expression at initial surgery increasing their surgical outcome from suboptimal $(>1 \mathrm{~cm})$ to optimal after SCS. Secondary eIF3a expression was not associated with residual tumor either (Table 2, $\mathrm{P}=0.660$ ). However, the difference in eIF3a expression between the two surgeries was associated with the surgical outcome of SCS. As shown in Table 3, more patients in non-decreased group of eIF3a enhanced their surgical outcome of secondary surgery, compared to those in decreased group of eIF3a $(60.9 \%$ vs. $0.0 \%, \mathrm{P}=0.041)$.

Expressions of HE4 and eIF3a at initial surgery did not show any difference among other clinicopathologic parameters including: FIGO stage, tumor grade, histological subtype, ascites, NACT, lymph node metastasis, menopause, and the cycles of two postoperative chemotherapies (data not shown, all $\mathrm{P}>0.05)$. For their expressions at secondary surgery, only HE4 expression was associated with tumor grade. The ratio of high HE4 expression decreased significantly in the sequence of borderline, G2, and G3 ovarian cancers: $6(100 \%)$ vs. 9 (75\%) vs. 2 $(33 \%), \mathrm{P}=0.036$.

\section{Correlations of HE4 and elF3a with survival of women with ROC}

HE4 expression at the time of both initial and secondary surgeries correlated with the overall survival of women with ROC. As shown in Figure 2A,B, patients with high HE4 expression at either surgery had better survival, compared to those with low HE4 expression [the median OS (95\% CI) for high vs. low expression at ICS, $127.8 \pm 28.3$ (72.4-183.2) vs. $36.0 \pm 5.2(25.8-46.2)$ months, $\mathrm{P}=0.011$; the median OS $(95 \% \mathrm{CI})$ for high $v$ s. low expression at SCS, $127.8 \pm$ 25.8 (77.3-178.3) vs. $49.3 \pm 19.8$ (10.5-88.0) months, $\mathrm{P}=$ 0.002]. Furthermore, patients with an elevated total score (TS) of HE4 between the two surgeries showed a trend towards a longer OS, compared to those with a non-elevated TS of HE4 [Figure 2C, the median OS
(95\% CI), $141.7 \pm 17.6$ (107.3-176.2) vs. $84.8 \pm 5.1$ (74.7-94.9) months, $\mathrm{P}=0.076]$. This result indicates that an elevated TS of HE4 could predict better survival in women with ROC, although the difference was not significant. HE4 expression at the time of both surgeries did not correlate with PFS.

eIF3a expression at the time of both surgeries did not correlate with PFS and OS either (data not shown, all $\mathrm{P}>0.05)$. However, the difference in eIF3a expression between the two surgeries correlated with OS. Patients who decreased the TS of eIF3a at SCS would prolong their OS, compared to those with a non-decreased TS of eIF3a [Figure 2D, the mean OS $(95 \% \mathrm{CI}), 133.4 \pm 22.0(90.2-176.6)$ vs. $87.3 \pm 9.6$ (68.4-106.2) months, $\mathrm{P}=0.052]$. Among our patients, there was only one woman who had the combination of an elevated TS of HE4 and a decreased TS of eIF3a, so we were not able to statistically analyze the combined effect of HE4 and eIF3a on survival. However, that woman has the longest OS in our study (Table 1, 150.5 months).

Next, we created a multivariable model that included following variables: initial and secondary HE4 expression (high vs. low), initial and secondary eIF3a expression (high vs. low), initial and secondary residual tumor $(0 \mathrm{~cm} v s .<1 \mathrm{~cm} v s .>1 \mathrm{~cm})$, histological subtype (serous vs. mucinous vs. others), tumor grade (borderline vs. G2 vs. G3), FIGO stage (I+II vs. III+IV), PFS, volume of ascites, menopause, NACT, lymph node metastasis, and the cycles of two postoperative chemotherapies. Initial HE4 expression was the only independent prognostic factor for OS (HR 8.91, 95\%CI 1.65-48.03, $\mathrm{P}=0.011$ ), as identified using the method of forward Stepwise (Likelihood Ratio).

\section{Discussion}

No data were previously reported about HE4 and eIF3a expressions in women with ROC who underwent SCS. To our knowledge, the present study revealed, for the first time, that eIF3a expression increases and HE4 expression is similar at the time of SCS, compared to the levels at initial surgery. We also demonstrated that HE4 and eIF3a expressions in paraffin-fixed ROC specimens are associated with surgical outcome, in terms of residual tumor, as well as OS in women with ROC.

Table 3. Associations of HE4 and elF3a expression with the surgical outcome of SCS.

\begin{tabular}{|c|c|c|c|c|}
\hline & & \multicolumn{3}{|c|}{ Surgical outcome of SCS } \\
\hline & & Enhanced n (\%) & Non-enhanced n (\%) & $P$ value \\
\hline \multirow[t]{2}{*}{ HE4 expression at secondary surgery } & Low expression & $5(29.4 \%)$ & $12(70.6 \%)$ & 0.038 \\
\hline & High expression & $7(77.8 \%)$ & $2(22.2 \%)$ & \\
\hline \multirow{2}{*}{ Difference in eIF3a expression between the two surgeries } & Decreased group & $0(0.0 \%)$ & $5(100.0 \%)$ & 0.041 \\
\hline & Non-decreased group & $14(60.9 \%)$ & $9(39.1 \%)$ & \\
\hline
\end{tabular}


A HE4 expression at initial surgery

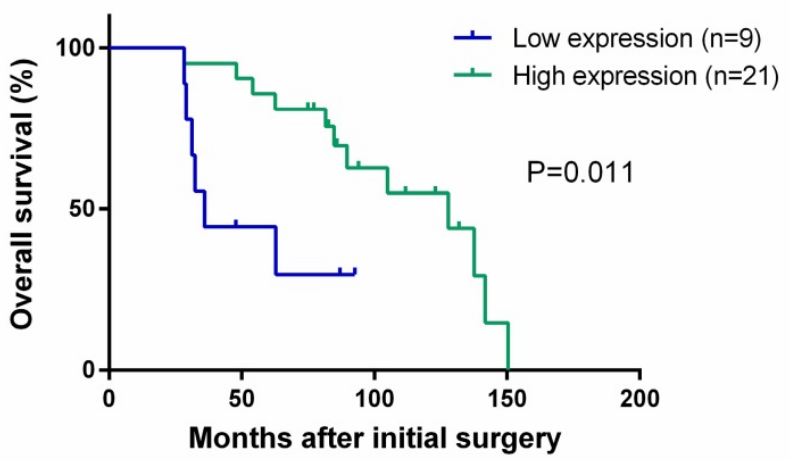

C Difference in HE4 expression

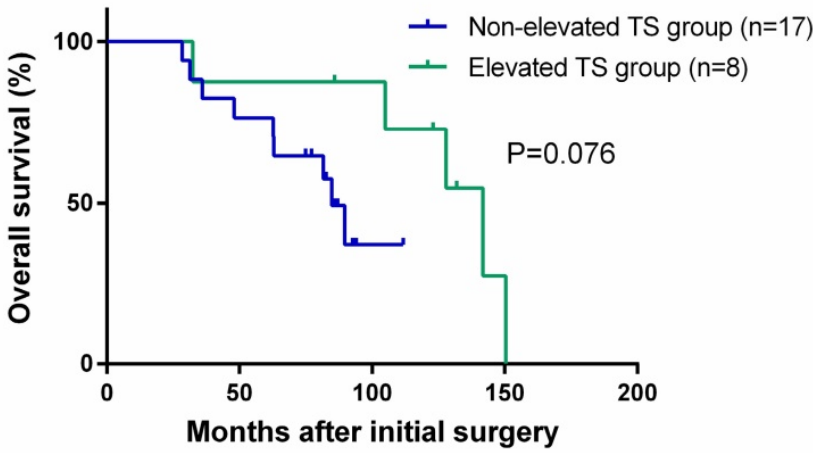

B HE4 expression at secondary surgery

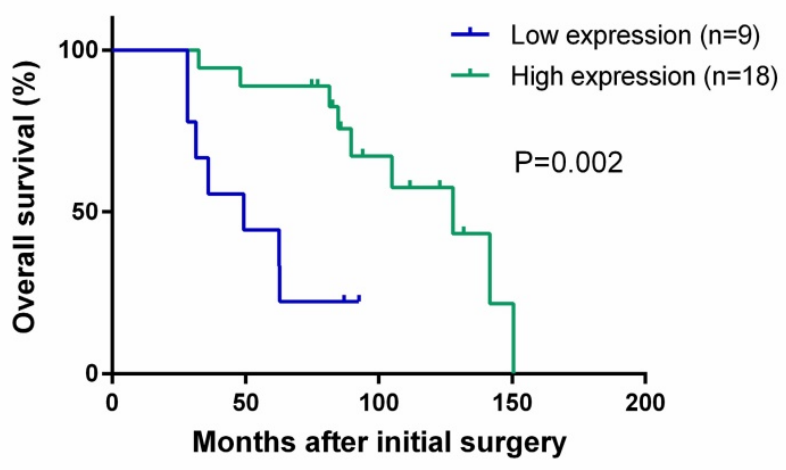

D Difference in elF3a expression

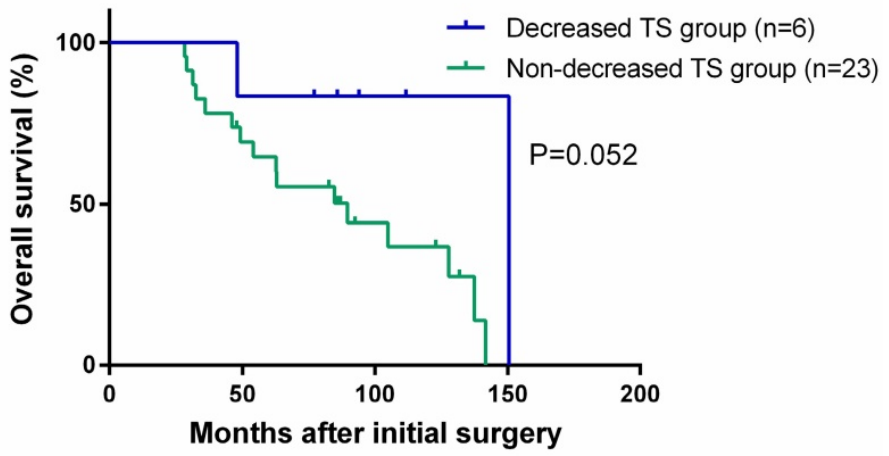

Figure 2. Correlation of HE4 and elF3a expressions with OS by using the Kaplan-Meier method and log-rank test. (A) HE4 expression at initial surgery correlated with OS. (B) HE4 expression at secondary surgery correlated with OS. (C) Patients with an elevated TS of HE4 between the two surgeries had prolonged OS. (D) Patients with a decreased TS of elF3a between the two surgeries had prolonged OS.

Braicu et al. was the first study group to report the association between plasma HE4 levels and surgical outcome in ROC patients[2]. Recently, by using serum HE4 levels, Angioli et al. proposed a new predictive Secondary Cytoreduction Score (SeC-S) to determine the probability of achieving optimal SCS[20]. However, no previous studies reported the association between surgical outcome and HE4 expression in tumor specimens of EOC. Our findings showed that high levels of HE4 in tumor specimens from ICS or SCS correlated with optimal surgical outcome.

Furthermore, our data showed that initial eIF3a expression was associated with secondary residual tumor. This means that initial eIF3a expression can predict the surgical outcome of secondary cytoreduction. Patients with low eIF3a expression at initial surgery were more likely to achieve optimal cytoreduction in SCS, compared to those with high eIF3a expression. Consequently, the elevated TS of eIF3a meant a higher probability of enhancing residual tumor after SCS. Further study is needed to validate the potential of eIF3a as a biomarker for identifying optimal candidates for SCS.
In previous studies, HE4 and eIF3a preoperative circulatory levels and their IHC staining levels in tumor specimens from ICS were always utilized to predict the prognosis of women with EOC[2, 5, 8, 14, $15,21]$. In the present study, our data indicated that the elevated TS of HE4 between ICS and SCS, as well as high HE4 expression at either surgery, also predicted a better overall survival in women with ROC. However, this prediction is not consistent with previous reports, in which high serum levels or high IHC staining levels of HE4 meant a worse survival in women with primary EOC[2, 8, 9]. Similarly, our results on the survival prediction of eIF3a expression differed from those of previous studies. Our results showed that decreasing the TS of eIF3a between the two surgeries indicated better survival in ROC, while previous studies reported that high eIF3a expression correlated with better survival in primary EOC[14]. Our own study on eIF3a expression in primary EOC also demonstrated that high eIF3a expression in tumor specimens correlated with better OS (unpublished data).

We assume that SCS is one possible reason for the above inconsistent results. Because the biggest difference between the previous studies and the 
present study is SCS: that is, ROC patients in the present study all underwent SCS but none of them did in previous studies. Numerous retrospective studies have validated that SCS significantly increases OS in both platinum-sensitive and platinum-resistant ROC[22-25]. For women with ROC, the strongest predictor of OS is having maximal cytoreduction with minimal residual tumor, at best, no residual tumor after SCS[24]. Therefore, SCS is recommended by the NCCN guidelines[1]. In the present study, high HE4 expression correlated with optimal cytoreduction. Also, patients with high HE4 expression at SCS were more likely to enhance their surgical outcome of secondary surgery. Even though the residual tumor itself was not associated with survival in the present study (data not shown), we still speculated that SCS had a superior power than HE4 on the survival of ROC patients and even reversed the impact of HE4 on survival. As a result, ROC patients with high HE4 expression presented prolonged OS in our study, which was inconsistent with previous studies in primary EOC patients.

Like HE4, similar effect of SCS on eIF3a was found in this study. Initial and secondary eIF3a expression was not associated with the corresponding residual tumor, thus eIF3a expression at the time of both surgeries did not correlate with PFS and OS. Additionally, due to SCS, the reduction in TS of eIF3a between the two surgeries indicated a better survival in ROC, which was also inconsistent with previous studies in primary EOC patients.

Certainly, other possible biological or pathophysiological mechanism for these inconsistent results might exist, which calls for further investigation. The evident limitations of our study are its retrospective character, the small sample size, and the lack of external validation. Therefore, larger studies should be performed. CA125 was reported as a predictor of surgical outcome and prognosis in women with EOC by previous studies[2, 26], but our data on CA125 was not comparable due to differences in methods for detecting CA125 among the patients. So CA125 was excluded in this study.

In conclusion, this study is a single-center study to retrospectively investigate the role of HE4 and eIF3a in predicting surgical outcome and overall survival in ROC patients with SCS. HE4 and eIF3a expressions in tumor specimens from ICS and SCS indicate a unique impact on surgical outcome and overall survival, respectively, and this impact differed from the effect of both serum and tissue levels of HE4 and EIF3a at the time of ICS. Further, larger studies are required to determine whether these impacts are independent or related to SCS.

\section{Abbreviations}

HE4: human epididymis protein 4; eIF3a: eukaryotic translation initiation factor 3a; EOC: epithelial ovarian cancer; ROC: recurrent ovarian cancer; ICS: initial cytoreductive surgery; SCS: secondary cytoreductive surgery; IHC: immunohistochemistry; TS: total score; KPS: Karnofsky Performance Status; FIGO: Federation of Gynecology and Obstetrics; OS: overall survival; PFS: progression free survival; NACT: neoadjuvant chemotherapy.

\section{Acknowledgements}

We thank Yue Zhou (Xiangya School of Medicine, Central South University, Changsha, Hunan, People's Republic of China) for revising this manuscript. This work was supported by the Key program of Hunan Provincial Science \& Technology Department (2013FJ2009 to C-H Luo); the Hunan Provincial Natural Science Foundation of China (2015JJ2093 to J Wang); the National Key R\&D Program of China (2016YFC1303703 to J Wang); and the National Natural Science Foundation of China (81522048).

\section{Competing Interests}

The authors have declared that no competing interest exists.

\section{References}

1. Morgan RJ, Jr., Armstrong DK, Alvarez RD, Bakkum-Gamez JN, Behbakht K, Chen LM, et al. Ovarian Cancer, Version 1.2016, NCCN Clinical Practice Guidelines in Oncology. J Natl Compr Canc Netw. 2016; 14: 1134-63.

2. Braicu EI, Chekerov R, Richter R, Pop C, Nassir M, Loefgren H, et al. HE4 expression in plasma correlates with surgical outcome and overall survival in patients with first ovarian cancer relapse. Ann Surg Oncol. 2014; 21: 955-62.

3. Hauspy J, Covens A. Cytoreductive surgery for recurrent ovarian cancer. Curr Opin Obstet Gynecol. 2007; 19: 15-21.

4. Hellstrom I, Raycraft J, Hayden-Ledbetter M, Ledbetter JA, Schummer M, McIntosh M, et al. The HE4 (WFDC2) protein is a biomarker for ovarian carcinoma. Cancer Res. 2003; 63: 3695-700.

5. Scaletta G, Plotti F, Luvero D, Capriglione S, Montera R, Miranda A, et al. The role of novel biomarker HE4 in the diagnosis, prognosis and follow-up of ovarian cancer: a systematic review. Expert Rev Anticancer Ther. 2017; 17: 827-39.

6. Romagnolo C, Leon AE, Fabricio ASC, Taborelli M, Polesel J, Del Pup L, et al. HE4, CA125 and risk of ovarian malignancy algorithm (ROMA) as diagnostic tools for ovarian cancer in patients with a pelvic mass: An Italian multicenter study. Gynecol Oncol. 2016; 141: 303-11.

7. Karlsen MA, Hogdall EV, Christensen II, Borgfeldt C, Kalapotharakos G, Zdrazilova-Dubska L, et al. A novel diagnostic index combining HE4, CA125 and age may improve triage of women with suspected ovarian cancer - An international multicenter study in women with an ovarian mass. Gynecol Oncol. 2015; 138: 640-6.

8. Zhu LC, Gao J, Hu ZH, Schwab CL, Zhuang HY, Tan MZ, et al. Membranous expressions of Lewis y and CAM-DR-related markers are independent factors of chemotherapy resistance and poor prognosis in epithelial ovarian cancer. Am J Cancer Res. 2015; 5: 830-43.

9. Moore RG, Hill EK, Horan T, Yano N, Kim K, MacLaughlan S, et al. HE4 (WFDC2) gene overexpression promotes ovarian tumor growth. Sci Rep. 2014; 4: 3574 .

10. Lee AS, Kranzusch PJ, Cate JH. eIF3 targets cell-proliferation messenger RNAs for translational activation or repression. Nature. 2015; 522: 111-4.

11. Harel-Sharvit L, Eldad N, Haimovich G, Barkai O, Duek L, Choder M. RNA polymerase II subunits link transcription and mRNA decay to translation. Cell. 2010; 143: 552-63.

12. Bolger TA, Folkmann AW, Tran EJ, Wente SR. The mRNA export factor Gle1 and inositol hexakisphosphate regulate distinct stages of translation. Cell. 2008; 134: 624-33. 
13. Isken O, Kim YK, Hosoda N, Mayeur GL, Hershey JW, Maquat LE. Upf1 phosphorylation triggers translational repression during nonsense-mediated mRNA decay. Cell. 2008; 133: 314-27.

14. Zhang Y, Yu JJ, Tian Y, Li ZZ, Zhang CY, Zhang SF, et al. eIF3a improve cisplatin sensitivity in ovarian cancer by regulating XPC and p27Kip1 translation. Oncotarget. 2015; 6: 25441-51.

15. Hershey JW. The role of eIF3 and its individual subunits in cancer. Biochim Biophys Acta. 2015; 1849: 792-800.

16. Spilka R, Ernst C, Mehta AK, Haybaeck J. Eukaryotic translation initiation factors in cancer development and progression. Cancer Lett. 2013; 340: 9-21.

17. Wang J, Luo C, Wang Y, Tang Y, Fang K, Zeng L, et al. [Correlation between eIF3a and HE4 expression and ovarian cancer]. Zhong Nan Da Xue Xue Bao Yi Xue Ban. 2014; 39: 1240-5.

18. Shen J, Yin JY, Li XP, Liu ZQ, Wang Y, Chen J, et al. The prognostic value of altered eIF3a and its association with p27 in non-small cell lung cancers. PLoS One. 2014; 9: e96008.

19. Luo C-H, Zhao M, Tang Y-X, Shahabi S, Fang K-N, Chen Y, et al. Increased HE4 mRNA Expression Correlates with High Level of eIF3a mRNA and Better Survival in Women with Epithelial Ovarian Cancer. J Cancer. 2018; 9: 1088-95.

20. Angioli R, Capriglione S, Aloisi A, Ricciardi R, Scaletta G, Lopez S, et al. A Predictive Score for Secondary Cytoreductive Surgery in Recurrent Ovarian Cancer (SeC-Score): A Single-Centre, Controlled Study for Preoperative Patient Selection. Ann Surg Oncol. 2015; 22: 4217-23.

21. Kong SY, Han MH, Yoo HJ, Hwang JH, Lim MC, Seo SS, et al. Serum HE4 level is an independent prognostic factor in epithelial ovarian cancer. Ann Surg Oncol. 2012; 19: 1707-12.

22. Musella A, Marchetti C, Palaia I, Perniola G, Giorgini M, Lecce F, et al. Secondary Cytoreduction in Platinum-Resistant Recurrent Ovarian Cancer: A Single-Institution Experience. Ann Surg Oncol. 2015; 22: 4211-6.

23. Al Rawahi T, Lopes AD, Bristow RE, Bryant A, Elattar A, Chattopadhyay S, et al. Surgical cytoreduction for recurrent epithelial ovarian cancer. Cochrane Database Syst Rev. 2013: CD008765.

24. Suh DH, Kim HS, Chang SJ, Bristow RE. Surgical management of recurrent ovarian cancer. Gynecol Oncol. 2016; 142: 357-67.

25. Petrillo M, Pedone Anchora L, Tortorella L, Fanfani F, Gallotta V, Pacciani M, et al. Secondary cytoreductive surgery in patients with isolated platinum-resistant recurrent ovarian cancer: a retrospective analysis. Gynecol Oncol. 2014; 134: 257-61.

26. Braicu EI, Fotopoulou C, Van Gorp T, Richter R, Chekerov R, Hall C, et al. Preoperative HE4 expression in plasma predicts surgical outcome in primary ovarian cancer patients: results from the OVCAD study. Gynecol Oncol. 2013; 128: $245-51$. 